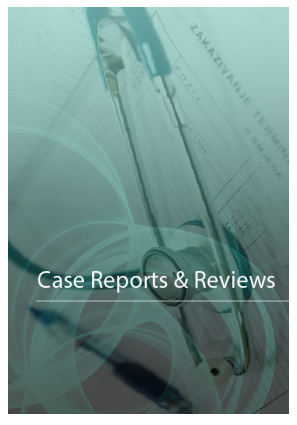

Correspondence

Dr. Maisano Roberto.

Medical Oncology Department, G.O.M. Reggio Calabria, Italy.

E-mail: robertomaisano@alice.it

- Received Date: 24 May 2020;

- Accepted Date: 06 Jun 2020;

- Publication Date: 09 Jun 2020.

Keywords

Epithelioid Sarcoma, Intestinal Invagination, Jejuno Neoplasm.

\section{Copyright}

(c) 2020 Science Excel. This is an openaccess article distributed under the terms of the Creative Commons Attribution 4.0 International license.

\title{
A very rare case of jejuno-jejunal invagination caused by epithelioid sarcoma: A case report and literature review
}

\author{
Maisano Roberto ${ }^{1 *}$, Azzarello Domenico', Fava Maria Giovanna ${ }^{2}$, Albonico \\ Giuseppe $^{3}$, Maisano Maurizio ${ }^{3}$, Mafodda Antonino ${ }^{1}$ and Agostino Rita Maria' \\ ${ }^{1}$ Medical Oncology Department, G.O.M. Reggio Calabria, Italy \\ ${ }^{2}$ General Surgery Department, G.O.M. Reggio Calabria, Italy \\ ${ }^{3}$ Pathological Anatomy Department, G.O.M. Reggio Calabria, Italy
}

\begin{abstract}
Rationale: Intestinal invagination or intussusception is a rare event in adult and it is linked to a specific pathology abnormality in $70 \%-90 \%$ of cases often a benign lesion of jejunal wall being malignant tumors rare.

Objective: We report our experience about a 71 year-old man presented to emergency with three days history of generalized abdominal pain and swelling, vomiting and partial bowel obstruction, ability to gas.

Findings: A total body computed tomography (CT) scan detected one thickened ansa in the proximal jejunum, which suggested an invagination. Moreover evident gastrectasis with duodenal fluid distension was reported. The patient underwent laparoscopy that revealed a strictured area of the jejunum with a mass lesion. Owing to technical difficulties during surgery, the procedure was converted to open laparotomy, that showed jejuno-jejunal invagination caused by an intestinal tumor. Reduction was impossible and resection was performed. Histopathological examination revealed a malignant neoplasm with the features of mesenchymal tumor. Malignant cells with pleomorphic and large vesicular nuclei, prominent nucleoli and ample of eosinophilic cytoplasm were described. Histochemical markers supported the diagnosis of epithelioid sarcoma. One month after surgery, TC scan was performed finding lung and liver metastases also confirmed at PET scan, therefore the patient received adriamycin chemotherapy without efficacy confirming poor prognosis of this tumor.

Conclusion: Being intussusceptions in adult a infrequent condition underlyning neoplastic cause should always ruled out.
\end{abstract}

\section{Introduction}

Intestinal invagination or intussusception is the leading cause of intestinal obstruction in children, but in adults it accounts for only 5\% of all intussusceptions, up to $5 \%$ of all cases of adult intestinal obstructions and $0.003 \%-0.02 \%$ of all adult hospital admissions. In contrast to childhood intussusception, which is idiopathic in $90 \%$ of cases, adult intussusceptions is linked to a specific cause which is pathologic abnormality in $70 \%-90 \%$ of cases, often a benign lesion of jejunal wall, being malignant neoplasms rare [1]. Sarcomas of the small bowel (SSB) are rare and represent around $8-15 \%$ of primary small bowel neoplasms [2]. Before the identification of gastrointestinal stromal tumors (GIST) as specific neoplasm, the majority of SSB were classified as leiomyoma, leiomyoblastoma or leiomysarcoma. Nowadays, GIST (85\%) are the most common gastrointestinal tract sarcomas followed by leiomyosarcoma. Others non-GIST sarcomas include liposarcoma, fibrosarcoma, Kaposi's sarcoma and angiosarcoma [3]. Epithelioid sarcoma (ES) is a distinct clinicopathologic entity. It is a rare soft tissue neoplasm of unknown histogenesis and usually occurs in the distal extremities of young adults [4].

\section{Case Presentation}

A 71 year-old Caucasian man presented to emergency department with three days history of generalized abdominal pain and swelling, vomiting and partial bowel obstruction, ability to gas. She reported abdominal pain, constipation and moderate weight loss for several months, but had not carried out any medical examination. A total body computed tomography (CT) scan detected one thickened ansa in the proximal jejunum, which suggested an invagination. Moreover evident gastrectasis with duodenal fluid distension was reported.

The patient underwent laparoscopy that revealed a strictured area of the jejunum with a mass lesion. Owing to technical difficulties during surgery, the 
procedure was converted to open laparotomy, that showed jejunojejunal invagination caused by an intestinal tumor. Reduction was impossible and resection was performed. Histopathological examination revealed a malignant neoplasm with the features of mesenchymal tumor. Malignant cells with pleomorphic and large vesicular nuclei, prominent nucleoli and ample of eosinophilic cytoplasm were described. Histochemical markers supported the diagnosis of ES (Table 1). One month after surgery, TC scan was performed finding lung and liver metastases also confirmed at PET scan, therefore the patient received adriamycin chemotherapy without benefit.

\begin{tabular}{|c|c|}
\hline Desmin & Absent \\
\hline S 100 & Absent \\
\hline CD 31 & Absent \\
\hline CD 34 & Absent \\
\hline CD 117 & Absent \\
\hline CD 68 & Absent \\
\hline Vimentin & Present \\
\hline MNF II6 & Present \\
\hline AE I/AE3 & Present \\
\hline CAM 5.2 & Present \\
\hline
\end{tabular}

Table 1: Immunohistochemical panel.

\section{Discussion}

In adult gastrointestinal invaginations are a rare events and the most common location on the small bowel is at distal end, ileo-ileal or ileocolic sites. Acute clinical presentation is characterized by complete mechanical obstruction and sometimes with strangulating injury. Conversely some patients report relapsing abdominal pain and hematochezia for a long time. Often, the diagnosis is achieved during laparotomy performed for acute symptoms. From $80 \%$ to $90 \%$ of all intestinal invaginations develop at the site of structural lesions of the bowel wall and about $15 \%$ of those are malignant tumors. Small bowel tumors are infrequent, about $3 \%$ of all gastrointestinal neoplasms and sarcomas are extremely rare. GIST are the most common followed by leiomyosarcoma. ES is a rare soft tissue sarcoma, described for the first time by Enzinger in 1970 [5]. Two variants are known, the conventional distal type often found in the distal extremities of young adults and the more recent proximal-type/axial type, described by Guillon in 1977 [6]. It affects older patients, mostly in axial or deep location and has an aggressive clinical behavior with poor outcome. Visceral location is extremely rare and is described in the colon, bladder and perineal sites. Radical surgery is the treatment of choice although the tendency to local relapse and pulmonary metastasis that worsen the prognosis.

\section{Conclusion}

Here, we presented an extremely rare case of small bowel intussusception linked to ES. Being intussusceptions in adult a infrequent condition underlyning neoplastic cause should always ruled out.

\section{References}

1. Andriessen MJ, Govaert MJ, de Waard JW. Jejunojejunal intussusceptions by a known jejunal adenocarcinoma. Can J Surg. 2008;51(4):83-84.

2. Bilimoria KY, Bentrem DJ, Wayne JD, Ko CY. Bennet CL, Talamonti MS. Small bowel cancer in the United States: changes in epidemiology, treatment and survival over last 20 years. Ann Surg. 2009;249:63-71.

3. Shenoy S. Small bowel sarcoma: tumor biology and advances in therapeutics. Surg Oncol. 2015;24(3):136-144.

4. Sobanko JF, Meijer L, Nigra TP. Epithelioid sarcoma: a review and update. J Aesthet Dermatol. 2009;2(5):49-54.

5. Enzinger FM. Epithelioid sarcoma. A sarcoma simulating a granuloma or a carcinoma. Cancer. 1970;26:1029-1041.

6. Guillou L, Wadden C, CoindreJM, Krausz T, Fletcher CD. "Proximal-type" epithelioid sarcoma, a distinctive aggressive neoplasm showing rhabdoid features. Clinicopathologic, immunohistochemical and ultrastructural study of a series. Am J Surg Pathol. 1997; 4: 491-495. 